\title{
Common fixed point results for four maps satisfying $\phi$-contractive condition in multiplicative metric spaces
}

\author{
Feng $\mathrm{Gu}^{1 *}$ and Yeol Je $\mathrm{Cho}^{2}$
}

\author{
"Correspondence: \\ gufeng99@sohu.com \\ ${ }^{1}$ Institute of Applied Mathematics, \\ Department of Mathematics, \\ Hangzhou Normal University, \\ Hangzhou, Zhejiang 310036, China \\ Full list of author information is \\ available at the end of the article
}

\begin{abstract}
In this paper, we consider the setting of multiplicative metric spaces to establish results regarding the common fixed points of four mappings, using a contraction condition defined by means of a comparison function. Also, we provide illustrative examples in support of our new results. The results obtained in this paper extend and improve some well-known comparable results in the literature due to He et al. (Fixed Point Theory and Appl. 2013:48, 2013).
\end{abstract}

MSC: $47 \mathrm{H} 10 ; 54 \mathrm{H} 25 ; 54 \mathrm{E} 50$

Keywords: multiplicative metric space; common fixed point; compatible mappings; weakly compatible mappings; comparison function

\section{Introduction and preliminaries}

The existence and uniqueness of fixed and common fixed point theorems of mappings has been a subject of great interest since Banach [1] proved the Banach contraction principle in 1922. In the past years, many authors generalized the Banach contraction principle in various spaces such as quasi-metric spaces, fuzzy metric spaces, 2-metric spaces, cone metric spaces, partial metric spaces and generalized metric spaces (see, for instance, [219] and the references therein). In 2008, Bashirov et al. [20] introduced the notion of multiplicative metric spaces, and studied the concept of multiplicative calculus and proved the fundamental theorem of multiplicative calculus. In 2012, Florack and Assen [21] displayed the use of the concept of multiplicative calculus in biomedical image analysis. In 2011, Bashirov et al. [22] exploit the efficiency of multiplicative calculus over the Newtonian calculus. They demonstrated that the multiplicative differential equations are more suitable than the ordinary differential equations in investigating some problems in various fields. Furthermore, Bashirov et al. [20] illustrated the usefulness of multiplicative calculus with some interesting applications. With the help of multiplicative absolute value function, they defined the multiplicative distance between two nonnegative real numbers as well as between two positive square matrices. This provides the basis for multiplicative metric spaces. In 2012, Özavşar and Çevikel [23] investigate multiplicative metric spaces by remarking its topological properties, and introduced concept of multiplicative contraction mapping and proved some fixed point theorems of multiplicative contraction mappings on

(c) $2015 \mathrm{Gu}$ and Cho. This article is distributed under the terms of the Creative Commons Attribution 4.0 International License (http://creativecommons.org/licenses/by/4.0/), which permits unrestricted use, distribution, and reproduction in any medium, provided you give appropriate credit to the original author(s) and the source, provide a link to the Creative Commons license, and indicate if changes were made. 
multiplicative spaces. Recently, He et al. [24] proved a common fixed point theorems for four self-mappings in multiplicative metric space. Very recently, Abbas et ai. [25] proved some common fixed point results of quasi-weak commutative mappings on a closed ball in the framework of multiplicative metric spaces. At the same time, they also studied the sufficient conditions for the existence of a common solution of multiplicative boundary value problem. Kang et al. [26] introduced the notions of compatible mappings and its variants in multiplicative metric spaces, and proved some common fixed point theorems for these mappings.

Now, we present some necessary definitions and results in multiplicative metric spaces, which will be needed in the sequel.

Definition 1.1 [20] Let $X$ be a nonempty set. A multiplicative metric is a mapping $d$ : $X \times X \rightarrow \mathbb{R}^{+}$satisfying the following axioms:

(M1) $d(x, y) \geq 1$ for all $x, y \in X$ and $d(x, y)=1 \Leftrightarrow x=y$;

(M2) $d(x, y)=d(y, x)$ for all $x, y \in X$;

(M3) $d(x, y) \leq d(x, z) \cdot d(z, y)$ for all $x, y, z \in X$ (multiplicative triangle inequality).

The pair $(X, d)$ is called a multiplicative metric space.

Definition 1.2 [20] Let $(X, d)$ be a multiplicative metric space, $\left\{x_{n}\right\}$ be a sequence in $X$ and $x \in X$. If for every multiplicative open ball $B_{\epsilon}(x)=\{y \mid d(x, y)<\epsilon\}, \epsilon>1$, there exists a natural number $N \in \mathbb{N}$ such that $n \geq N$, then $x_{n} \in B_{\epsilon}(x)$. Then the sequence $\left\{x_{n}\right\}$ is said to be multiplicative converging to $x$, denoted by $x_{n} \rightarrow x(n \rightarrow \infty)$.

Proposition 1.1 [23] Let $(X, d)$ be a multiplicative metric space, $\left\{x_{n}\right\}$ be a sequence in $X$ and $x \in X$. Then

$$
x_{n} \rightarrow x \quad(n \rightarrow \infty) \text { if and only if } \quad d\left(x_{n}, x\right) \rightarrow 1 \quad(n \rightarrow \infty) .
$$

Definition 1.3 [23] Let $(X, d)$ be a multiplicative metric space, $\left\{x_{n}\right\}$ be a sequence in $X$. The sequence $\left\{x_{n}\right\}$ is called multiplicative Cauchy sequence if, for each $\epsilon>0$, there exists a positive integer $N \in \mathbb{N}$ such that $d\left(x_{n}, x_{m}\right)<\epsilon$ for all $n, m \geq N$.

Proposition 1.2 [23] Let $(X, d)$ be a multiplicative metric space and $\left\{x_{n}\right\}$ be a sequence in X. Then $\left\{x_{n}\right\}$ is a multiplicative Cauchy sequence if and only if $d\left(x_{n}, x_{m}\right) \rightarrow 1(n, m \rightarrow \infty)$.

Definition 1.4 [23] A multiplicative metric space $(X, d)$ is said to be multiplicative complete if every multiplicative Cauchy sequence in $(X, d)$ is multiplicative convergent in $X$.

Definition 1.5 [23] Let $\left(X, d_{X}\right)$ and $\left(Y, d_{Y}\right)$ be two multiplicative metric spaces and $f$ : $X \rightarrow Y$ be a function. If for $f$ holds the requirement that, for every $\epsilon>1$, there exists $\delta>1$ such that $f\left(B_{\delta}(x)\right) \subset B_{\delta}(f(x))$, then we call $f$ multiplicative continuous at $x \in X$.

Proposition 1.3 [23] Let $\left(X, d_{X}\right)$ and $\left(Y, d_{Y}\right)$ be two multiplicative metric spaces, $f: X \rightarrow$ $Y$ be a mapping and $\left\{x_{n}\right\}$ be any sequence in $X$. Then $f$ is multiplicative continuous at $x \in X$ if and only if $f\left(x_{n}\right) \rightarrow f(x)$ for every sequence $\left\{x_{n}\right\}$ with $x_{n} \rightarrow x(n \rightarrow \infty)$. 
Proposition 1.4 [23] Let $\left(X, d_{X}\right)$ be a multiplicative metric space, $\left\{x_{n}\right\}$ and $\left\{y_{n}\right\}$ be two sequences in $X$ such that $x_{n} \rightarrow x, y_{n} \rightarrow y(n \rightarrow \infty), x, y \in X$. Then

$$
d\left(x_{n}, y_{n}\right) \rightarrow d(x, y) \quad(n \rightarrow \infty)
$$

Definition 1.6 The self-maps $f$ and $g$ of a set $X$ are called commutative if $f g x=g f x$ for all $x \in X$.

Definition 1.7 Suppose that $f, g$ are two self-mappings of a multiplicative metric space $(X, d)$. The pair $(f, g)$ are called weak commutative mappings if $d(f g x, g f x) \leq d(f x, g x)$ for all $x \in X$.

Definition 1.8 [23] Let $(X, d)$ be a multiplicative metric space, and let $f: X \rightarrow X$ be called a multiplicative contraction if there exists a real constant $\lambda \in(0,1]$ such that $d(f x, f y) \leq$ $d(x, y)^{\lambda}$ for all $x, y \in X$.

Theorem 1.1 [23] Let $(X, d)$ be a multiplicative metric space, and let $f: X \rightarrow X$ be a multiplicative contraction. If $(X, d)$ is complete, then $f$ has a unique fixed point.

Recently, He et al. [24] proved the following interesting theorems.

Theorem 1.2 [24] Let S, T, A, and B be self-mappings of a multiplicative metric space $X$, they satisfy the following conditions:

(i) $S X \subset B X, T X \subset A X$;

(ii) $A$ and $S$ are weak commutative, $B$ and $T$ also are weak commutative;

(iii) one of $S, T, A$, and $B$ is continuous;

(iv) $d(S x, T y) \leq\{\max \{d(A x, B y), d(A x, S x), d(B y, T y), d(S x, B y), d(A x, T y)\}\}^{\lambda}, \lambda \in\left(0, \frac{1}{2}\right)$, for all $x, y \in X$.

Then $S, T, A$, and $B$ have a unique common fixed point.

\section{Main results}

We start our work by introducing the following two concepts.

Definition 2.1 The self-maps $f$ and $g$ of a multiplicative metric space $(X, d)$ are said to be compatible if $\lim _{n \rightarrow \infty} d\left(f g x_{n}, g f x_{n}\right)=1$, whenever $\left\{x_{n}\right\}$ is a sequence in $X$ such that $\lim _{n \rightarrow \infty} f x_{n}=\lim _{n \rightarrow \infty} g x_{n}=t$, for some $t \in X$.

Definition 2.2 Suppose that $f$ and $g$ are two self-maps of a multiplicative metric space $(X, d)$. The pair $(f, g)$ are called weakly compatible mappings if $f x=g x, x \in X$ implies $f g x=$ $g f x$. That is, $d(f x, g x)=1 \Rightarrow d(f g x, g f x)=1$.

Remark 2.1 Commutative mappings must be weak commutative mappings, weak commutative mappings must be compatible, compatible mappings must be weakly compatible, but the converse is not true.

Example 2.1 Let $X=\mathbb{R}$ and $(X, d)$ be a multiplicative metric space defined by $d(x, y)=$ $e^{|x-y|}$ for all $x, y$ in $X$. Let $f$ and $g$ be two self-mappings defined by $f x=x^{3}, g x=2-x$. Then

$$
d\left(f x_{n}, g x_{n}\right)=e^{\left|x_{n}-1\right| \cdot\left|x_{n}^{2}+x_{n}+2\right|} \rightarrow 1 \quad \text { iff } \quad x_{n} \rightarrow 1
$$


and

$$
\lim _{n \rightarrow \infty} d\left(f g x_{n}, g f x_{n}\right)=\lim _{n \rightarrow \infty} e^{6\left|x_{n}-1\right|^{2}}=1 \quad \text { if } x_{n} \rightarrow 1
$$

Thus $f$ and $g$ are compatible. Note that

$$
d(f g 0, g f 0)=d(8,2)=e^{6}>e^{2}=d(0,2)=d(f 0, g 0),
$$

so the pair $(f, g)$ is not weakly commuting.

Example 2.2 Let $X=[0,+\infty),(X, d)$ be a multiplicative metric space defined by $d(x, y)=$ $e^{|x-y|}$ for all $x, y$ in $X$. Let $f$ and $g$ be two self-mappings defined by

$$
f x=\left\{\begin{array}{ll}
x, & \text { if } 0 \leq x<2, \\
2, & \text { if } x=2, \\
4, & \text { if } 2<x<+\infty,
\end{array} \quad g x= \begin{cases}4-x, & \text { if } 0 \leq x<2, \\
2, & \text { if } x=2, \\
7, & \text { if } 2<x<+\infty\end{cases}\right.
$$

By the definition of the mappings of $f$ and $g$, only for $x=2, f x=g x=2$, at this time $f g x=$ $g f x=2$, so we see the pair $(f, g)$ is weakly compatible.

For $x_{n}=2-\frac{1}{n} \in(0,2)$, from the definition of the mappings of $f$ and $g$ we have

$$
\lim _{n \rightarrow \infty} f x_{n}=\lim _{n \rightarrow \infty} g x_{n}=2,
$$

but

$$
\lim _{n \rightarrow \infty} d\left(f g x_{n}, g f x_{n}\right)=\lim _{n \rightarrow \infty} e^{x_{n}}=e^{2} \neq 1,
$$

so the pair $(f, g)$ is not compatible.

Let $\Phi$ denote the set of functions $\phi:[1, \infty)^{5} \rightarrow[0, \infty)$ satisfying

(1) $\phi$ is nondecreasing and continuous in each coordinate variable;

(2) for $t \geq 1$,

$$
\psi(t)=\max \{\phi(t, t, t, 1, t), \phi(t, t, t, t, 1), \phi(t, 1,1, t, t), \phi(1, t, 1, t, 1), \phi(1,1, t, 1, t)\} \leq t
$$

From now on, unless otherwise stated, we choose $\phi \in \Phi$.

Theorem 2.1 Let $(X, d)$ be a complete multiplicative metric space, $S, T, A$, and $B$ be four mappings of $X$ into itself. Suppose that there exists $\lambda \in\left(0, \frac{1}{2}\right)$ such that $S(X) \subset B(X), T(X) \subset$ $A(X)$, and

$$
d(S x, T y) \leq \phi\left(d^{\lambda}(A x, B y), d^{\lambda}(A x, S x), d^{\lambda}(B y, T y), d^{\lambda}(S x, B y), d^{\lambda}(A x, T y)\right)
$$

for all $x, y \in X$. Assume one of the following conditions is satisfied:

(a) either $A$ or $S$ is continuous, the pair $(S, A)$ is compatible and the pair $(T, B)$ is weakly compatible; 
(b) either $B$ or $T$ is continuous, the pair $(T, B)$ is compatible and the pair $(S, A)$ is weakly compatible.

Then $S, T, A$, and B have a unique common fixed point.

Proof Let $x_{0} \in X$. Since $S(X) \subset B(X)$ and $T(X) \subset A(X)$, there exist $x_{1}, x_{2} \in X$ such that $y_{0}=S x_{0}=B x_{1}$ and $y_{1}=T x_{1}=A x_{2}$. By induction, there exist sequences $\left\{x_{n}\right\}$ and $\left\{y_{n}\right\}$ in $X$ such that

$$
y_{2 n}=S x_{2 n}=B x_{2 n+1}, \quad y_{2 n+1}=T x_{2 n+1}=A x_{2 n+2}
$$

for all $n=0,1,2, \ldots$.

Next, we prove that $\left\{y_{n}\right\}$ is a multiplicative Cauchy sequence in $X$. In fact, $\forall n \in \mathbb{N}$, from (2.1), (2.2), and the property of $\psi$ we have

$$
\begin{aligned}
d\left(y_{2 n}, y_{2 n+1}\right)= & d\left(S x_{2 n}, T x_{2 n+1}\right) \\
\leq & \phi\left(d^{\lambda}\left(A x_{2 n}, B x_{2 n+1}\right), d^{\lambda}\left(A x_{2 n}, S x_{2 n}\right), d^{\lambda}\left(B x_{2 n+1}, T x_{2 n+1}\right),\right. \\
& \left.d^{\lambda}\left(S x_{2 n}, B x_{2 n+1}\right), d^{\lambda}\left(A x_{2 n}, T x_{2 n+1}\right)\right) \\
= & \phi\left(d^{\lambda}\left(y_{2 n-1}, y_{2 n}\right), d^{\lambda}\left(y_{2 n-1}, y_{2 n}\right), d^{\lambda}\left(y_{2 n}, y_{2 n+1}\right),\right. \\
& \left.d^{\lambda}\left(y_{2 n}, y_{2 n}\right), d^{\lambda}\left(y_{2 n-1}, y_{2 n+1}\right)\right) \\
\leq & \phi\left(d^{\lambda}\left(y_{2 n-1}, y_{2 n}\right), d^{\lambda}\left(y_{2 n-1}, y_{2 n}\right), d^{\lambda}\left(y_{2 n}, y_{2 n+1}\right), 1,\right. \\
& \left.d^{\lambda}\left(y_{2 n-1}, y_{2 n}\right) \cdot d^{\lambda}\left(y_{2 n}, y_{2 n+1}\right)\right) \\
\leq & \phi\left(d^{\lambda}\left(y_{2 n-1}, y_{2 n}\right) \cdot d^{\lambda}\left(y_{2 n}, y_{2 n+1}\right), d^{\lambda}\left(y_{2 n-1}, y_{2 n}\right) \cdot d^{\lambda}\left(y_{2 n}, y_{2 n+1}\right),\right. \\
& \left.d^{\lambda}\left(y_{2 n-1}, y_{2 n}\right) \cdot d^{\lambda}\left(y_{2 n}, y_{2 n+1}\right), 1, d^{\lambda}\left(y_{2 n-1}, y_{2 n}\right) \cdot d^{\lambda}\left(y_{2 n}, y_{2 n+1}\right)\right) \\
\leq & \psi\left(d^{\lambda}\left(y_{2 n-1}, y_{2 n}\right) \cdot d^{\lambda}\left(y_{2 n}, y_{2 n+1}\right)\right) \\
\leq & d^{\lambda}\left(y_{2 n-1}, y_{2 n}\right) \cdot d^{\lambda}\left(y_{2 n}, y_{2 n+1}\right) .
\end{aligned}
$$

This implies that

$$
d\left(y_{2 n}, y_{2 n+1}\right) \leq d^{\frac{\lambda}{1-\lambda}}\left(y_{2 n-1}, y_{2 n}\right)=d^{h}\left(y_{2 n-1}, y_{2 n}\right)
$$

Here $h=\frac{\lambda}{1-\lambda} \in(0,1)$.

Similarly, using (2.1), (2.2), and the property of $\psi$, we have

$$
\begin{aligned}
d\left(y_{2 n+1}, y_{2 n+2}\right)= & d\left(T x_{2 n+1}, S x_{2 n+2}\right)=d\left(S x_{2 n+2}, T x_{2 n+1}\right) \\
\leq & \phi\left(d^{\lambda}\left(A x_{2 n+2}, B x_{2 n+1}\right), d^{\lambda}\left(A x_{2 n+2}, S x_{2 n+2}\right), d^{\lambda}\left(B x_{2 n+1}, T x_{2 n+1}\right)\right. \\
& \left.d^{\lambda}\left(S x_{2 n+2}, B x_{2 n+1}\right), d^{\lambda}\left(A x_{2 n+2}, T x_{2 n+1}\right)\right) \\
= & \phi\left(d^{\lambda}\left(y_{2 n+1}, y_{2 n}\right), d^{\lambda}\left(y_{2 n+1}, y_{2 n+2}\right), d^{\lambda}\left(y_{2 n}, y_{2 n+1}\right)\right. \\
& \left.d^{\lambda}\left(y_{2 n+2}, y_{2 n}\right), d^{\lambda}\left(y_{2 n+1}, y_{2 n+1}\right)\right) \\
\leq & \phi\left(d^{\lambda}\left(y_{2 n}, y_{2 n+1}\right), d^{\lambda}\left(y_{2 n+1}, y_{2 n+2}\right), d^{\lambda}\left(y_{2 n}, y_{2 n+1}\right)\right. \\
& \left.d^{\lambda}\left(y_{2 n}, y_{2 n+1}\right) \cdot d^{\lambda}\left(y_{2 n+1}, y_{2 n+2}\right), 1\right)
\end{aligned}
$$




$$
\begin{aligned}
\leq & \phi\left(d^{\lambda}\left(y_{2 n}, y_{2 n+1}\right) \cdot d^{\lambda}\left(y_{2 n+1}, y_{2 n+2}\right), d^{\lambda}\left(y_{2 n}, y_{2 n+1}\right) \cdot d^{\lambda}\left(y_{2 n+1}, y_{2 n+2}\right),\right. \\
& \left.d^{\lambda}\left(y_{2 n}, y_{2 n+1}\right) \cdot d^{\lambda}\left(y_{2 n+1}, y_{2 n+2}\right), d^{\lambda}\left(y_{2 n}, y_{2 n+1}\right) \cdot d^{\lambda}\left(y_{2 n+1}, y_{2 n+2}\right), 1\right) \\
\leq & \psi\left(d^{\lambda}\left(y_{2 n}, y_{2 n+1}\right) \cdot d^{\lambda}\left(y_{2 n+1}, y_{2 n+2}\right)\right) \\
\leq & d^{\lambda}\left(y_{2 n}, y_{2 n+1}\right) \cdot d^{\lambda}\left(y_{2 n+1}, y_{2 n+2}\right) .
\end{aligned}
$$

This implies that

$$
d\left(y_{2 n+1}, y_{2 n+2}\right) \leq d^{\frac{\lambda}{1-\lambda}}\left(y_{2 n}, y_{2 n+1}\right)=d^{h}\left(y_{2 n}, y_{2 n+1}\right) .
$$

It follows from (2.3) and (2.4) that, for all $n \in \mathbb{N}$, we have

$$
d\left(y_{n}, y_{n+1}\right) \leq d^{h}\left(y_{n-1}, y_{n}\right) \leq d^{h^{2}}\left(y_{n-2}, y_{n-1}\right) \leq \cdots \leq d^{h^{n}}\left(y_{0}, y_{1}\right) .
$$

Therefore, for all $n, m \in \mathbb{N}, n<m$, by the multiplicative triangle inequality we obtain

$$
\begin{aligned}
d\left(y_{n}, y_{m}\right) & \leq d\left(y_{n}, y_{n+1}\right) \cdot d\left(y_{n+1}, y_{n+2}\right) \cdots \cdot d\left(y_{m-1}, y_{m}\right) \\
& \leq d^{h^{n}}\left(y_{0}, y_{1}\right) \cdot d^{h^{n+1}}\left(y_{0}, y_{1}\right) \cdots \cdot d^{h^{m-1}}\left(y_{0}, y_{1}\right) \\
& \leq d^{\frac{h^{n}}{1-h}}\left(y_{0}, y_{1}\right) .
\end{aligned}
$$

This implies that $d\left(y_{n}, y_{m}\right) \rightarrow 1(n, m \rightarrow \infty)$. Hence $\left\{y_{n}\right\}$ is a multiplicative Cauchy sequence in $X$. By the completeness of $X$, there exists $z \in X$ such that $y_{n} \rightarrow z(n \rightarrow \infty)$.

Moreover, because

$$
\left\{y_{2 n}\right\}=\left\{S x_{2 n}\right\}=\left\{B x_{2 n+1}\right\} \quad \text { and } \quad\left\{y_{2 n+1}\right\}=\left\{T x_{2 n+1}\right\}=\left\{A x_{2 n+2}\right\}
$$

are subsequences of $\left\{y_{n}\right\}$, we obtain

$$
\lim _{n \rightarrow \infty} S x_{2 n}=\lim _{n \rightarrow \infty} B x_{2 n+1}=\lim _{n \rightarrow \infty} T x_{2 n+1}=\lim _{n \rightarrow \infty} A x_{2 n+2}=z .
$$

Next, we prove $z$ is a common fixed point of $S, T, A$, and $B$ under the condition (a).

Case 1. Suppose that $A$ is a continuous, then $\lim _{n \rightarrow \infty} A S x_{2 n}=\lim _{n \rightarrow \infty} A^{2} x_{2 n}=A z$. Since the pair $(S, A)$ is compatible, from (2.5) we have

$$
\lim _{n \rightarrow \infty} d\left(S A x_{2 n}, A S x_{2 n}\right)=\lim _{n \rightarrow \infty} d\left(S A x_{2 n}, A z\right)=1,
$$

that is, $\lim _{n \rightarrow \infty} S A x_{2 n}=A z$. By using (2.1) and (2.2) we have

$$
\begin{aligned}
d\left(S A x_{2 n}, T x_{2 n+1}\right) \leq & \phi\left(d^{\lambda}\left(A^{2} x_{2 n}, B x_{2 n+1}\right), d^{\lambda}\left(A^{2} x_{2 n}, S A x_{2 n}\right), d^{\lambda}\left(B x_{2 n+1}, T x_{2 n+1}\right),\right. \\
& \left.d^{\lambda}\left(S A x_{2 n}, B x_{2 n+1}\right), d^{\lambda}\left(A^{2} x_{2 n}, T x_{2 n+1}\right)\right) .
\end{aligned}
$$

Taking $n \rightarrow \infty$ on the two sides of the above inequality, using (2.5) and the property of $\psi$, we get

$$
\begin{aligned}
d(A z, z) & \leq \phi\left(d^{\lambda}(A z, z), d^{\lambda}(A z, A z), d^{\lambda}(z, z), d^{\lambda}(A z, z), d^{\lambda}(A z, z)\right) \\
& =\phi\left(d^{\lambda}(A z, z), 1,1, d^{\lambda}(A z, z), d^{\lambda}(A z, z)\right)
\end{aligned}
$$




$$
\begin{aligned}
& \leq \psi\left(d^{\lambda}(A z, z)\right) \\
& \leq d^{\lambda}(A z, z) .
\end{aligned}
$$

This means that $d(A z, z)=1$, that is, $A z=z$. Again applying (2.1) and (2.2), we obtain

$$
\begin{gathered}
d\left(S z, T x_{2 n+1}\right) \leq \phi\left(d^{\lambda}\left(A z, B x_{2 n+1}\right), d^{\lambda}(A z, S z), d^{\lambda}\left(B x_{2 n+1}, T x_{2 n+1}\right),\right. \\
\left.d^{\lambda}\left(S z, B x_{2 n+1}\right), d^{\lambda}\left(A z, T x_{2 n+1}\right)\right) .
\end{gathered}
$$

Letting $n \rightarrow \infty$ on both sides in the above inequality, using $A z=z$, (2.5), and the property of $\psi$, we can obtain

$$
\begin{aligned}
d(S z, z) & \leq \phi\left(d^{\lambda}(z, z), d^{\lambda}(z, S z), d^{\lambda}(z, z), d^{\lambda}(S z, z), d^{\lambda}(z, z)\right) \\
& =\phi\left(1, d^{\lambda}(S z, z), 1, d^{\lambda}(S z, z), 1\right) \\
& \leq \psi\left(d^{\lambda}(S z, z)\right) \\
& \leq d^{\lambda}(S z, z) .
\end{aligned}
$$

This implies that $d(S z, z)=1$, that is, $S z=z$.

On the other hand, since $z=S z \in S X \subset B X$, there exists $z^{*} \in X$ such that $z=S z=B z^{*}$. By using (2.1), $z=S z=A z=B z^{*}$, and the property of $\psi$, we can obtain

$$
\begin{aligned}
d\left(z, T z^{*}\right) & =d\left(S z, T z^{*}\right) \\
& \leq \phi\left(d^{\lambda}\left(A z, B z^{*}\right), d^{\lambda}(A z, S z), d^{\lambda}\left(B z^{*}, T z^{*}\right), d^{\lambda}\left(S z, B z^{*}\right), d^{\lambda}\left(A z, T z^{*}\right)\right) \\
& =\phi\left(d^{\lambda}(z, z), d^{\lambda}(z, z), d^{\lambda}\left(z, T z^{*}\right), d^{\lambda}(z, z), d^{\lambda}\left(z, T z^{*}\right)\right) \\
& =\phi\left(1,1, d^{\lambda}\left(z, T z^{*}\right), 1, d^{\lambda}\left(z, T z^{*}\right)\right) \\
& \leq \psi\left(d^{\lambda}\left(z, T z^{*}\right)\right) \\
& \leq d^{\lambda}\left(z, T z^{*}\right) .
\end{aligned}
$$

This implies that $d\left(z, T z^{*}\right)=1$, and so $T z^{*}=z=B z^{*}$. Since the pair $(T, B)$ is weakly compatible, we have

$$
T z=T B z^{*}=B T z^{*}=B z .
$$

Now we prove that $T z=z$. From (2.1) and the property of $\psi$, we have

$$
\begin{aligned}
d(z, T z) & =d(S z, T z) \\
& \leq \phi\left(d^{\lambda}(A z, B z), d^{\lambda}(A z, S z), d^{\lambda}(B z, T z), d^{\lambda}(S z, B z), d^{\lambda}(A z, T z)\right) \\
& =\phi\left(d^{\lambda}(z, T z), d^{\lambda}(z, z), d^{\lambda}(T z, T z), d^{\lambda}(z, T z), d^{\lambda}(z, T z)\right) \\
& =\phi\left(d^{\lambda}(z, T z), 1,1, d^{\lambda}(z, T z), d^{\lambda}(z, T z)\right) \\
& \leq \psi\left(d^{\lambda}(z, T z)\right) \\
& \leq d^{\lambda}(z, T z)
\end{aligned}
$$

This implies that $d(z, T z)=1$, so $z=T z$. 
Therefore, we obtain $z=S z=A z=T z=B z$, so $z$ is a common fixed point of $S, T, A$, and $B$.

Case 2. Suppose that $S$ is continuous, then $\lim _{n \rightarrow \infty} S A x_{2 n}=\lim _{n \rightarrow \infty} S^{2} x_{2 n}=S z$. Since the pair $(S, A)$ is compatible, from (2.5) we have

$$
\lim _{n \rightarrow \infty} d\left(S A x_{2 n}, A S x_{2 n}\right)=\lim _{n \rightarrow \infty} d\left(S z, A S x_{2 n}\right)=1,
$$

that is, $\lim _{n \rightarrow \infty} A S x_{2 n}=S z$. From (2.1) and (2.2) we obtain

$$
\begin{aligned}
d\left(S^{2} x_{2 n}, T x_{2 n+1}\right) \leq & \phi\left(d^{\lambda}\left(A S x_{2 n}, B x_{2 n+1}\right), d^{\lambda}\left(A S x_{2 n}, S^{2} x_{2 n}\right), d^{\lambda}\left(B x_{2 n+1}, T x_{2 n+1}\right),\right. \\
& \left.d^{\lambda}\left(S^{2} x_{2 n}, B x_{2 n+1}\right), d^{\lambda}\left(A S x_{2 n}, T x_{2 n+1}\right)\right) .
\end{aligned}
$$

Taking $n \rightarrow \infty$ on the two sides of the above inequality, using (2.5) and the property of $\psi$, we can get

$$
\begin{aligned}
d(S z, z) & \leq \phi\left(d^{\lambda}(S z, z), d^{\lambda}(S z, S z), d^{\lambda}(z, z), d^{\lambda}(S z, z), d^{\lambda}(S z, z)\right) \\
& =\phi\left(d^{\lambda}(S z, z), 1,1, d^{\lambda}(S z, z), d^{\lambda}(S z, z)\right) \\
& \leq \psi\left(d^{\lambda}(S z, z)\right) \\
& \leq d^{\lambda}(S z, z) .
\end{aligned}
$$

This means that $d(S z, z)=1$, this is $S z=z$.

Since $z=S z \in S X \subset B X$, there exists $z^{*} \in X$ such that $z=S z=B z^{*}$. From (2.1) we have

$$
\begin{aligned}
d\left(S^{2} x_{2 n}, T z^{*}\right) \leq & \phi\left(d^{\lambda}\left(A S x_{2 n}, B z^{*}\right), d^{\lambda}\left(A S x_{2 n}, S^{2} x_{2 n}\right), d^{\lambda}\left(B z^{*}, T z^{*}\right),\right. \\
& \left.d^{\lambda}\left(S^{2} x_{2 n}, B z^{*}\right), d^{\lambda}\left(A S x_{2 n}, T z^{*}\right)\right) .
\end{aligned}
$$

Letting $n \rightarrow \infty$, using $z=S z=B z^{*}$ and the property of $\psi$, we can obtain

$$
\begin{aligned}
d\left(z, T z^{*}\right) & \leq \phi\left(d^{\lambda}\left(S z, B z^{*}\right), d^{\lambda}(S z, S z), d^{\lambda}\left(z, T z^{*}\right), d^{\lambda}(S z, z), d^{\lambda}\left(S z, T z^{*}\right)\right) \\
& =\phi\left(d^{\lambda}(z, z), d^{\lambda}(z, z), d^{\lambda}\left(z, T z^{*}\right), d^{\lambda}(z, z), d^{\lambda}\left(z, T z^{*}\right)\right) \\
& =\phi\left(1,1, d^{\lambda}\left(z, T z^{*}\right), 1, d^{\lambda}\left(z, T z^{*}\right)\right) \\
& \leq \psi\left(d^{\lambda}\left(z, T z^{*}\right)\right) \\
& \leq d^{\lambda}\left(z, T z^{*}\right) .
\end{aligned}
$$

This implies that $d\left(z, T z^{*}\right)=1$, and so $T z^{*}=z=B z^{*}$. Since the pair $(T, B)$ is weakly compatible, we obtain

$$
T z=T B z^{*}=B T z^{*}=B z .
$$

So $T z=B z$. By $(2.1)$ and the property of $\psi$, we have

$$
\begin{gathered}
d\left(S x_{2 n}, T z\right) \leq \\
d^{\lambda}\left(S x_{2 n}^{\lambda}\left(A x_{2 n}, B z\right), d^{\lambda}\left(A x_{2 n}, T z\right)\right) .
\end{gathered}
$$


Taking $n \rightarrow \infty$ on the two sides of the above inequality, using $B z=T z$ and the property of $\psi$, we can get

$$
\begin{aligned}
d(z, T z) & \leq \phi\left(d^{\lambda}(z, B z), d^{\lambda}(z, z), d^{\lambda}(B z, T z), d^{\lambda}(z, B z), d^{\lambda}(z, T z)\right) \\
& =\phi\left(d^{\lambda}(z, T z), d^{\lambda}(z, z), d^{\lambda}(T z, T z), d^{\lambda}(z, T z), d^{\lambda}(z, T z)\right) \\
& =\phi\left(d^{\lambda}(z, T z), 1,1, d^{\lambda}(z, T z), d^{\lambda}(z, T z)\right) \\
& \leq \psi\left(d^{\lambda}(z, T z)\right) \\
& \leq d^{\lambda}(z, T z) .
\end{aligned}
$$

This implies that $d(z, T z)=1$, so $z=T z=B z$.

On the other hand, since $z=T z \in T X \subset A X$, there exists $z^{* *} \in X$ such that $z=T z=A z^{* *}$. By (2.1), using $T z=B z=z$ and the property of $\psi$, we can obtain

$$
\begin{aligned}
d\left(S z^{* *}, z\right) & =d\left(S z^{* *}, T z\right) \\
& \leq \phi\left(d^{\lambda}\left(A z^{* *}, B z\right), d^{\lambda}\left(A z^{* *}, S z^{* *}\right), d^{\lambda}(B z, T z), d^{\lambda}\left(S z^{* *}, B z\right), d^{\lambda}\left(A z^{* *}, T z\right)\right) \\
& =\phi\left(d^{\lambda}(z, z), d^{\lambda}\left(z, S z^{* *}\right), d^{\lambda}(z, z), d^{\lambda}\left(S z^{* *}, z\right), d^{\lambda}(z, z)\right) \\
& =\phi\left(1, d^{\lambda}\left(S z^{* *}, z\right), 1, d^{\lambda}\left(S z^{* *}, z\right), 1\right) \\
& \leq \psi\left(d^{\lambda}\left(S z^{* *}, z\right)\right) \\
& \leq d^{\lambda}\left(S z^{* *}, z\right) .
\end{aligned}
$$

This implies that $d\left(S z^{* *}, z\right)=1$, and so $S z^{* *}=z=A z^{* *}$.

Since the pair $(S, A)$ is compatible,

$$
d(S z, A z)=d\left(S A z^{* *}, A S z^{* *}\right)=d(z, z)=1 .
$$

So $A z=S z$. Hence $z=S z=A z=T z=B z$.

Next, we prove that $S, T, A$, and $B$ have a unique common fixed point. Suppose that $w \in X$ is also a common fixed point of $S, T, A$ and $B$, then

$$
\begin{aligned}
d(z, w) & =d(S z, T w) \\
& \leq \phi\left(d^{\lambda}(A z, B w), d^{\lambda}(A z, S z), d^{\lambda}(B w, T w), d^{\lambda}(S z, B w), d^{\lambda}(A z, T w)\right) \\
& =\phi\left(d^{\lambda}(z, w), d^{\lambda}(z, z), d^{\lambda}(w, w), d^{\lambda}(z, w), d^{\lambda}(z, w)\right) \\
& =\phi\left(d^{\lambda}(z, w), 1,1, d^{\lambda}(z, w), d^{\lambda}(z, w)\right) \\
& \leq \psi\left(d^{\lambda}(z, w)\right) \\
& \leq d^{\lambda}(z, w) .
\end{aligned}
$$

This implies that $d(z, w)=1$, and so $w=z$. Therefore, $z$ is a unique common fixed point of $S, T, A$, and $B$.

Finally, if condition (b) holds, then the argument is similar to that above, so we delete it. This completes the proof. 
Remark 2.2 Theorem 2.1 generalizes and extends the corresponding results of He et al. [24], Theorem 3.1 and Kang et al. [26], Theorem 3.2.

Example 2.3 Let $X=[0,2]$, and $(X, d)$ be a multiplicative metric space defined by $d(x, y)=$ $e^{|x-y|}$ for all $x, y$ in $X$. Let $S, T, A$, and $B$ be four self-mappings defined by

$$
\begin{aligned}
& S x=\frac{5}{4}, \quad \forall x \in[0,2], \quad T x= \begin{cases}\frac{7}{4}, & x \in[0,1], \\
\frac{5}{4}, & x \in(1,2],\end{cases} \\
& A x=\left\{\begin{array}{ll}
1, & x \in[0,1], \\
\frac{5}{4}, & x \in(1,2), \\
\frac{7}{4}, & x=2,
\end{array} \quad B x= \begin{cases}\frac{1}{4}, & x \in[0,1], \\
\frac{5}{4}, & x \in(1,2), \\
1, & x=2 .\end{cases} \right.
\end{aligned}
$$

Note that $S$ is multiplicative continuous in $X$, and $T, A$, and $B$ are not multiplicative continuous mappings in $X$.

(i) Clearly we can get $S(X) \subset B(X)$ and $T(X) \subset A(X)$.

(ii) By the definition of the mappings of $S$ and $A$, only for $\left\{x_{n}\right\} \subset(1,2)$, we have

$$
\lim _{n \rightarrow \infty} S x_{n}=\lim _{n \rightarrow \infty} A x_{n}=t=\frac{5}{4} .
$$

At this time

$$
\lim _{n \rightarrow \infty} d\left(S A x_{n}, A S_{n} x\right)=d\left(\frac{5}{4}, \frac{5}{4}\right)=1,
$$

so we can see the pair $(S, A)$ is compatible.

By the definition of the mappings of $T$ and $B$, only for $x \in(1,2), T x=B x=\frac{5}{4}, T B x=$ $T\left(\frac{5}{4}\right)=\frac{5}{4}=B\left(\frac{5}{4}\right)=B T x$, so $T B x=B T x$, thus we can see the pair $(T, B)$ to be weakly compatible.

(iii) Now we prove that the mappings $S, T, A$, and $B$ satisfy the condition (2.1) of Theorem 2.1 with $\lambda=\frac{2}{3}$ and $\phi\left(t_{1}, t_{2}, t_{3}, t_{4}, t_{5}\right)=\frac{1}{5}\left(t_{1}+t_{2}+t_{3}+t_{4}+t_{5}\right)$. For this, we consider the following cases:

Case 1. If $x, y \in[0,1]$, then

$$
d(S x, T y)=d\left(\frac{5}{4}, \frac{7}{4}\right)=e^{\frac{1}{2}}
$$

and

$$
\begin{aligned}
\phi & \left(d^{\lambda}(A x, B y), d^{\lambda}(A x, S x), d^{\lambda}(B y, T y), d^{\lambda}(S x, B y), d^{\lambda}(A x, T y)\right) \\
& =\phi\left(d^{\frac{2}{3}}\left(1, \frac{1}{4}\right), d^{\frac{2}{3}}\left(1, \frac{5}{4}\right), d^{\frac{2}{3}}\left(\frac{1}{4}, \frac{7}{4}\right), d^{\frac{2}{3}}\left(\frac{5}{4}, \frac{1}{4}\right), d^{\frac{2}{3}}\left(1, \frac{7}{4}\right)\right) \\
& =\phi\left(e^{\frac{1}{2}}, e^{\frac{1}{6}}, e, e^{\frac{2}{3}}, e\right) \\
& =\frac{1}{5}\left(e^{\frac{1}{2}}+e^{\frac{1}{6}}+e+e^{\frac{2}{3}}+e\right) \\
& =e^{\frac{1}{2}} \cdot \frac{1}{5}\left(1+e^{-\frac{1}{3}}+e^{\frac{1}{2}}+e^{\frac{1}{6}}+e^{\frac{1}{2}}\right) \\
& >e^{\frac{1}{2}} .
\end{aligned}
$$


Thus we have

$d(S x, T y)=e^{\frac{1}{2}}<\phi\left(d^{\lambda}(A x, B y), d^{\lambda}(A x, S x), d^{\lambda}(B y, T y), d^{\lambda}(S x, B y), d^{\lambda}(A x, T y)\right)$.

Case 2. If $x \in[0,1], y \in(1,2]$, then we obtain

$$
\begin{aligned}
d(S x, T y) & =d\left(\frac{5}{4}, \frac{5}{4}\right) \\
& =1 \leq \phi\left(d^{\lambda}(A x, B y), d^{\lambda}(A x, S x), d^{\lambda}(B y, T y), d^{\lambda}(S x, B y), d^{\lambda}(A x, T y)\right) .
\end{aligned}
$$

Case 3. If $x \in(1,2), y \in[0,1]$, then

$$
d(S x, T y)=d\left(\frac{5}{4}, \frac{7}{4}\right)=e^{\frac{1}{2}}
$$

and

$$
\begin{aligned}
\phi & \left(d^{\lambda}(A x, B y), d^{\lambda}(A x, S x), d^{\lambda}(B y, T y), d^{\lambda}(S x, B y), d^{\lambda}(A x, T y)\right) \\
& =\phi\left(d^{\frac{2}{3}}\left(\frac{5}{4}, \frac{1}{4}\right), d^{\frac{2}{3}}\left(\frac{5}{4}, \frac{5}{4}\right), d^{\frac{2}{3}}\left(\frac{1}{4}, \frac{7}{4}\right), d^{\frac{2}{3}}\left(\frac{5}{4}, \frac{1}{4}\right), d^{\frac{2}{3}}\left(\frac{5}{4}, \frac{7}{4}\right)\right) \\
& =\phi\left(e^{\frac{2}{3}}, 1, e, e^{\frac{2}{3}}, e^{\frac{1}{3}}\right) \\
& =\frac{1}{5}\left(e^{\frac{2}{3}}, 1, e, e^{\frac{2}{3}}, e^{\frac{1}{3}}\right) \\
& =e^{\frac{1}{2}} \cdot \frac{1}{5}\left(e^{\frac{1}{6}}+e^{-\frac{1}{2}}+e^{\frac{1}{2}}+e^{\frac{1}{6}}+e^{-\frac{1}{6}}\right) \\
& >e^{\frac{1}{2}}
\end{aligned}
$$

Hence we have

$d(S x, T y)=e^{\frac{1}{2}}<\phi\left(d^{\lambda}(A x, B y), d^{\lambda}(A x, S x), d^{\lambda}(B y, T y), d^{\lambda}(S x, B y), d^{\lambda}(A x, T y)\right)$.

Case 4 . If $x=2, y \in[0,1]$, then

$$
d(S x, T y)=d\left(\frac{5}{4}, \frac{7}{4}\right)=e^{\frac{1}{2}}
$$

and

$$
\begin{aligned}
\phi & \left(d^{\lambda}(A x, B y), d^{\lambda}(A x, S x), d^{\lambda}(B y, T y), d^{\lambda}(S x, B y), d^{\lambda}(A x, T y)\right) \\
& =\phi\left(d^{\frac{2}{3}}\left(\frac{7}{4}, \frac{1}{4}\right), d^{\frac{2}{3}}\left(\frac{7}{4}, \frac{5}{4}\right), d^{\frac{2}{3}}\left(\frac{1}{4}, \frac{7}{4}\right), d^{\frac{2}{3}}\left(\frac{5}{4}, \frac{1}{4}\right), d^{\frac{2}{3}}\left(\frac{7}{4}, \frac{7}{4}\right)\right) \\
& =\phi\left(e, e^{\frac{1}{3}}, e, e^{\frac{2}{3}}, 1\right) \\
& =\frac{1}{5}\left(e+e^{\frac{1}{3}}+e+e^{\frac{2}{3}}+1\right) \\
& =e^{\frac{1}{2}} \cdot \frac{1}{5}\left(e^{\frac{1}{2}}+e^{-\frac{1}{6}}+e^{\frac{1}{2}}+e^{\frac{1}{6}}+e^{-\frac{1}{2}}\right) \\
& >e^{\frac{1}{2}} .
\end{aligned}
$$


Hence we have

$$
d(S x, T y)=e^{\frac{1}{2}}<\phi\left(d^{\lambda}(A x, B y), d^{\lambda}(A x, S x), d^{\lambda}(B y, T y), d^{\lambda}(S x, B y), d^{\lambda}(A x, T y)\right) .
$$

Case 5. If $x, y \in(1,2]$, then

$$
\begin{aligned}
d(S x, T y) & =d\left(\frac{5}{4}, \frac{5}{4}\right) \\
& =1 \leq \phi\left(d^{\lambda}(A x, B y), d^{\lambda}(A x, S x), d^{\lambda}(B y, T y), d^{\lambda}(S x, B y), d^{\lambda}(A x, T y)\right) .
\end{aligned}
$$

Then in all the above cases, the mappings $S, T, A$, and $B$ satisfy the condition (2.1) of Theorem 2.1 with $\lambda=\frac{2}{3}$ and $\phi\left(t_{1}, t_{2}, t_{3}, t_{4}, t_{5}\right)=\frac{1}{5}\left(t_{1}+t_{2}+t_{3}+t_{4}+t_{5}\right)$. So all the conditions of Theorem 2.1 are satisfied. Moreover, $\frac{5}{4}$ is the unique common fixed point for all of the mappings $S, T, A$, and $B$.

Theorem 2.2 Let $(X, d)$ be a complete multiplicative metric space, $S, T, A$, and $B$ be four mappings of $X$ into itself. Suppose that there exist $\lambda \in\left(0, \frac{1}{2}\right)$ and $p, q \in \mathbb{Z}^{+}$such that $S(X) \subset$ $B(X), T(X) \subset A(X)$, and

$$
d\left(S^{p} x, T^{q} y\right) \leq \phi\left(d^{\lambda}(A x, B y), d^{\lambda}\left(A x, S^{p} x\right), d^{\lambda}\left(B y, T^{q} y\right), d^{\lambda}\left(S^{p} x, B y\right), d^{\lambda}\left(A x, T^{q} y\right)\right)
$$

for all $x, y \in X$. Assume the following conditions are satisfied:

(a) the pairs $(S, A)$ and $(T, B)$ are commutative mappings;

(b) one of $S, T, A$, and $B$ is continuous.

Then $S, T, A$, and $B$ have a unique common fixed point.

Proof From $S(X) \subset B(X), T(X) \subset A(X)$ we have

$$
S^{p} X \subset S^{p-1} X \subset \cdots \subset S^{2} X \subset S X \subset B X
$$

and

$$
T^{q} X \subset T^{q-1} X \subset \cdots \subset T^{2} X \subset T X \subset A X
$$

Since the pairs $(S, A)$ and $(T, B)$ are commutative mappings,

$$
S^{p} A=S^{p-1} S A=S^{p-1} A S=S^{p-2}(S A) S=S^{p-2} A S^{2}=\cdots=A S^{p}
$$

and

$$
T^{q} B=T^{q-1} T B=T^{q-1} B T=T^{q-2}(T B) T=T^{q-2} B T^{2}=\cdots=B T^{q} .
$$

That is to say, $S^{p} A=A S^{p}$ and $T^{q} B=B T^{q}$.

It follows from Remark 2.1 that the pairs $\left(S^{p}, A\right)$ and $\left(T^{q}, B\right)$ are compatible and also weakly compatible. Therefore, by Theorem 2.1 , we can find that $S^{p}, T^{q}, A$, and $B$ have a unique common fixed point $z$. 
In addition, we prove that $S, T, A$, and $B$ have a unique common fixed point. From (2.6) and the property of $\psi$ we have

$$
\begin{aligned}
d(S z, z) & =d\left(S^{p}(S z), T^{q} z\right) \\
& \leq \phi\left(d^{\lambda}(A S z, B z), d^{\lambda}\left(A S z, S^{p}(S z)\right), d^{\lambda}\left(B z, T^{q} z\right), d^{\lambda}\left(S^{p}(S z), B z\right), d^{\lambda}\left(A S z, T^{q} z\right)\right) \\
& =\phi\left(d^{\lambda}(S z, z), d^{\lambda}(S z, S z), d^{\lambda}(z, z), d^{\lambda}(S z, z), d^{\lambda}(S z, z)\right) \\
& =\phi\left(d^{\lambda}(S z, z), 1,1, d^{\lambda}(S z, z), d^{\lambda}(S z, z)\right) \\
& \leq \psi\left(d^{\lambda}(S z, z)\right) \\
& \leq d^{\lambda}(S z, z) .
\end{aligned}
$$

This implies that $d(S z, z)=1$, so $S z=z$.

On the other hand, we have

$$
\begin{aligned}
d(z, T z) & =d\left(S^{p} z, T^{q}(T z)\right) \\
& \leq \phi\left(d^{\lambda}(A z, B T z), d^{\lambda}\left(A z, S^{p} z\right), d^{\lambda}\left(B T z, T^{q}(T z)\right), d^{\lambda}\left(S^{p} z, B T z\right), d^{\lambda}\left(A z, T^{q}(T z)\right)\right) \\
& =\phi\left(d^{\lambda}(S z, z), d^{\lambda}(z, z), d^{\lambda}(T z, T z), d^{\lambda}(z, T z), d^{\lambda}(z, T z)\right) \\
& =\phi\left(d^{\lambda}(S z, z), 1,1, d^{\lambda}(z, T z), d^{\lambda}(z, T z)\right) \\
& \leq \psi\left(d^{\lambda}(z, T z)\right) \\
& \leq d^{\lambda}(z, T z) .
\end{aligned}
$$

This implies that $d(z, T z)=1$, i.e., $T z=z$.

Therefore, we obtain $S z=T z=A z=B z=z$, so $z$ is a common fixed point of $S, T, A$, and $B$.

Finally, we prove that $S, T, A$, and $B$ have a unique common fixed point. Suppose that $w \in X$ is also a common fixed point of $S, T, A$ and $B$, then

$$
\begin{aligned}
d(z, w) & =d\left(S^{p} z, T^{q} w\right) \\
& \leq \phi\left(d^{\lambda}(A z, B w), d^{\lambda}\left(A z, S^{p} z\right), d^{\lambda}\left(B w, T^{q} w\right), d^{\lambda}\left(S^{p} z, B w\right), d^{\lambda}\left(A z, T^{q} w\right)\right) \\
& =\phi\left(d^{\lambda}(z, w), d^{\lambda}(z, z), d^{\lambda}(w, w), d^{\lambda}(z, w), d^{\lambda}(z, w)\right) \\
& =\phi\left(d^{\lambda}(z, w), 1,1, d^{\lambda}(z, w), d^{\lambda}(z, w)\right) \\
& \leq \psi\left(d^{\lambda}(z, w)\right) \\
& \leq d^{\lambda}(z, w) .
\end{aligned}
$$

This implies that $d(z, w)=1$, and so $w=z$. Therefore, $z$ is a unique common fixed point of $S, T, A$, and $B$.

Remark 2.3 Theorem 2.2 generalizes and extends the corresponding results of He et al. [24], Theorem 3.2.

By taking $\phi\left(t_{1}, t_{2}, t_{3}, t_{4}, t_{5}\right)=\max \left\{t_{1}, t_{2}, t_{3}, t_{4}, t_{5}\right\}$ in Theorems 2.1 and 2.2 , we have the following results. 
Corollary 2.1 Let $(X, d)$ be a complete multiplicative metric space, $S, T, A$, and $B$ be four mappings of $X$ into itself. Suppose that there exists $\lambda \in\left(0, \frac{1}{2}\right)$ such that $S(X) \subset B(X), T(X) \subset$ $A(X)$, and

$$
d(S x, T y) \leq \max \left\{d^{\lambda}(A x, B y), d^{\lambda}(A x, S x), d^{\lambda}(B y, T y), d^{\lambda}(S x, B y), d^{\lambda}(A x, T y)\right\}
$$

for all $x, y \in X$. Assume one of the following conditions is satisfied:

(a) either $A$ or $S$ is continuous, the pair $(S, A)$ is compatible and the pair $(T, B)$ is weakly compatible;

(b) either $B$ or $T$ is continuous, the pair $(T, B)$ is compatible and the pair $(S, A)$ is weakly compatible.

Then $S, T, A$, and $B$ have a unique common fixed point.

Remark 2.4 Theorem 3.1 of [24] is a special case of Corollary 2.1. Corollary 2.1 also improves and extends Theorem 3.2 of Kang et al. [26].

Corollary 2.2 Let $(X, d)$ be a complete multiplicative metric space, $S, T, A$, and B be four mappings of $X$ into itself. Suppose that there exist $\lambda \in\left(0, \frac{1}{2}\right)$ and $p, q \in \mathbb{Z}^{+}$such that $S(X) \subset$ $B(X), T(X) \subset A(X)$, and

$$
\begin{aligned}
d\left(S^{p} x, T^{q} y\right) \leq & \max \left\{d^{\lambda}(A x, B y), d^{\lambda}\left(A x, S^{p} x\right), d^{\lambda}\left(B y, T^{q} y\right),\right. \\
& \left.d^{\lambda}\left(S^{p} x, B y\right), d^{\lambda}\left(A x, T^{q} y\right)\right\}
\end{aligned}
$$

for all $x, y \in X$. Assume the following conditions are satisfied:

(a) the pairs $(S, A)$ and $(T, B)$ are commutative mappings;

(b) one of $S, T, A$, and $B$ is continuous.

Then $S, T, A$, and $B$ have a unique common fixed point.

Remark 2.5 Theorem 3.2 of [24] is a special case of Corollary 2.2.

Now we introduce an example to support Corollary 2.1.

Example 2.4 Let $X=[0,2]$, and $(X, d)$ be a multiplicative metric space defined by $d(x, y)=$ $e^{|x-y|}$ for all $x, y$ in $X$. Let $S, T, A$, and $B$ be four self-mappings defined by

$$
\begin{aligned}
& S x=\frac{7}{6}, \quad \forall x \in[0,2], \quad T x= \begin{cases}\frac{3}{2}, & x \in[0,1], \\
\frac{7}{6}, & x \in(1,2],\end{cases} \\
& A x=\left\{\begin{array}{ll}
1, & x \in[0,1], \\
\frac{7}{6}, & x \in(1,2), \\
\frac{3}{2}, & x=2,
\end{array} \quad B x= \begin{cases}\frac{1}{6}, & x \in[0,1], \\
\frac{7}{6}, & x \in(1,2), \\
1, & x=2 .\end{cases} \right.
\end{aligned}
$$

Clearly we can get $S(X) \subset B(X)$ and $T(X) \subset A(X)$.

Note that $T, A$, and $B$ are not multiplicative continuous mappings, and $S$ is multiplicative continuous in $X$.

By the definition of the mappings of $S$ and $A$, we have

$$
d(S A x, A S x)=d\left(\frac{7}{6}, \frac{7}{6}\right)=1 \leq d(S x, A x),
$$


which implies that the pair $(S, A)$ is weak commutative. Therefore, the pair $(S, A)$ must be compatible.

Clearly, only for $x \in(1,2), T x=B x=\frac{7}{6}, T B x=T\left(\frac{7}{6}\right)=\frac{7}{6}=B\left(\frac{7}{6}\right)=B T x$, so $T B x=B T x$, thus we can see the pair $(T, B)$ to be weakly compatible.

Now we prove that the mappings $S, T, A$, and $B$ satisfy the condition (2.7) of Corollary 2.1 with $\lambda=\frac{2}{3}$. For this, we let

$$
M(x, y)=\max \left\{d^{\frac{2}{3}}(A x, B y), d^{\frac{2}{3}}(A x, S x), d^{\frac{2}{3}}(B y, T y), d^{\frac{2}{3}}(S x, B y), d^{\frac{2}{3}}(A x, T y)\right\} .
$$

Case 1. If $x, y \in[0,1]$, then we have

$$
d(S x, T y)=d\left(\frac{7}{6}, \frac{3}{2}\right)=e^{\frac{1}{3}}<e^{\frac{4}{3} \cdot \frac{2}{3}}=d^{\frac{2}{3}}\left(\frac{1}{6}, \frac{3}{2}\right)=d^{\frac{2}{3}}(B y, T y) \leq M(x, y) .
$$

Case 2. If $x \in[0,1], y \in(1,2]$, then

$$
d(S x, T y)=d\left(\frac{7}{6}, \frac{7}{6}\right)=1 \leq M(x, y) .
$$

Case 3. If $x \in(1,2], y \in[0,1]$, then

$$
d(S x, T y)=d\left(\frac{7}{6}, \frac{3}{2}\right)=e^{\frac{1}{3}}<e^{\frac{4}{3} \cdot \frac{2}{3}}=d^{\frac{2}{3}}\left(\frac{1}{6}, \frac{3}{2}\right)=d^{\frac{2}{3}}(B y, T y) \leq M(x, y) .
$$

Case 4. If $x, y \in(1,2]$, then

$$
d(S x, T y)=d\left(\frac{7}{6}, \frac{7}{6}\right)=1 \leq M(x, y) .
$$

Then in all the above cases, the mappings $S, T, A$, and $B$ satisfy the condition (2.7) of Corollary 2.1 with $\lambda=\frac{2}{3}$. So all the conditions of Corollary 2.1 are satisfied. Moreover, $\frac{7}{6}$ is the unique common fixed point for all of the mappings $S, T, A$, and $B$.

Remark 2.6 Note that Example 2.4 satisfies all the hypotheses of Corollary 2.1. But Example 2.4 does not satisfy the hypotheses of Theorem 1.2. In fact, since

$$
d(T B 2, B T 2)=d\left(T 1, B\left(\frac{7}{6}\right)\right)=d\left(\frac{3}{2}, \frac{7}{6}\right)=e^{\frac{1}{3}}
$$

and

$$
d(T 2, B 2)=d\left(\frac{7}{6}, 1\right)=e^{\frac{1}{6}} .
$$

Thus $d(T B 2, B T 2)>d(T 2, B 2)$, and so the pair $(T, B)$ is not weak commutative.

Corollary 2.3 Let $(X, d)$ be a complete multiplicative metric space, $S, T, A$, and B be four mappings of $X$ into itself. Suppose that there exists $\lambda \in\left(0, \frac{1}{2}\right)$ such that $S(X) \subset B(X), T(X) \subset$ $A(X)$, and

$$
\begin{aligned}
d(S x, T y) \leq & a_{1} d^{\lambda}(A x, B y)+a_{2} d^{\lambda}(A x, S x) \\
& +a_{3} d^{\lambda}(B y, T y)+a_{4} d^{\lambda}(S x, B y)+a_{5} d^{\lambda}(A x, T y)
\end{aligned}
$$


for all $x, y \in X$. Here $a_{1}, a_{2}, a_{3}, a_{4}, a_{5} \geq 0$ and $0<a_{1}+a_{2}+a_{3}+a_{4}+a_{5} \leq 1$. Assume one of the following conditions is satisfied:

(a) either $A$ or $S$ is continuous, the pair $(S, A)$ is compatible and the pair $(T, B)$ is weakly compatible;

(b) either $B$ or $T$ is continuous, the pair $(T, B)$ is compatible and the pair $(S, A)$ is weakly compatible.

Then $S, T, A$, and B have a unique common fixed point.

Proof Suppose the condition (2.9) hold. For $x, y, z \in X$, let

$$
M(x, y, z)=\max \left\{d^{\lambda}(A x, B y), d^{\lambda}(A x, S x), d^{\lambda}(B y, T y), d^{\lambda}(S x, B y), d^{\lambda}(A x, T y)\right\} .
$$

Then

$$
\begin{aligned}
& a_{1} d^{\lambda}(A x, B y)+a_{2} d^{\lambda}(A x, S x)+a_{3} d^{\lambda}(B y, T y)+a_{4} d^{\lambda}(S x, B y)+a_{5} d^{\lambda}(A x, T y) \\
& \quad \leq\left(a_{1}+a_{2}+a_{3}+a_{4}+a_{5}\right) M(x, y, z) \\
& \quad \leq M(x, y, z)
\end{aligned}
$$

So, if (2.9) holds, then $d(S x, T y) \leq M(x, y, z)$ for all $x, y, z \in X$. Then the conclusion of Corollary 2.2 can be obtained from Corollary 2.1 immediately.

Corollary 2.4 Let $(X, d)$ be a complete multiplicative metric space, $S, T, A$, and $B$ be four mappings of $X$ into itself. Suppose that there exist $\lambda \in\left(0, \frac{1}{2}\right)$ and $p, q \in \mathbb{Z}^{+}$such that $S(X) \subset$ $B(X), T(X) \subset A(X)$ and

$$
\begin{aligned}
d\left(S^{p} x, T^{q} y\right) \leq & a_{1} d^{\lambda}(A x, B y)+a_{2} d^{\lambda}\left(A x, S^{p} x\right) \\
& +a_{3} d^{\lambda}\left(B y, T^{q} y\right)+a_{4} d^{\lambda}\left(S^{p} x, B y\right)+a_{5} d^{\lambda}\left(A x, T^{q} y\right)
\end{aligned}
$$

for all $x, y \in X$. Here $a_{1}, a_{2}, a_{3}, a_{4}, a_{5} \geq 0$ and $0<a_{1}+a_{2}+a_{3}+a_{4}+a_{5} \leq 1$. Assume the following conditions are satisfied:

(a) the pairs $(S, A)$ and $(T, B)$ are commutative mappings;

(b) one of $S, T, A$, and $B$ is continuous.

Then $S, T, A$, and B have a unique common fixed point.

Proof It is similar to the proof of Theorem 2.2.

By taking $A=B=I$ (the identity mappings) in Theorems 2.1 and 2.2, and Corollaries 2.1 and 2.2, we have the following results.

Corollary 2.5 Let $(X, d)$ be a complete multiplicative metric space, $S$ and $T$ be two mappings of $X$ into itself. Suppose that there exists $\lambda \in\left(0, \frac{1}{2}\right)$ such that

$$
d(S x, T y) \leq \phi\left(d^{\lambda}(x, y), d^{\lambda}(x, S x), d^{\lambda}(y, T y), d^{\lambda}(S x, y), d^{\lambda}(x, T y)\right)
$$

for all $x, y \in X$. Then $S$ and $T$ have a unique common fixed point. 
Corollary 2.6 Let $(X, d)$ be a complete multiplicative metric space, $S$ and $T$ be two mappings of $X$ into itself. Suppose that there exist $\lambda \in\left(0, \frac{1}{2}\right)$ and $p, q \in \mathbb{Z}^{+}$such that

$$
d\left(S^{p} x, T^{q} y\right) \leq \phi\left(d^{\lambda}(x, y), d^{\lambda}\left(x, S^{p} x\right), d^{\lambda}\left(y, T^{q} y\right), d^{\lambda}\left(S^{p} x, y\right), d^{\lambda}\left(x, T^{q} y\right)\right)
$$

for all $x, y \in X$. Then $S$ and $T$ have a unique common fixed point.

Corollary 2.7 Let $(X, d)$ be a complete multiplicative metric space, $S$ and $T$ be two mappings of $X$ into itself. Suppose that there exists $\lambda \in\left(0, \frac{1}{2}\right)$ such that

$$
d(S x, T y) \leq \max \left\{d^{\lambda}(x, y), d^{\lambda}(x, S x), d^{\lambda}(y, T y), d^{\lambda}(S x, y), d^{\lambda}(x, T y)\right\}
$$

for all $x, y \in X$. Then $S$ and $T$ have a unique common fixed point.

Corollary 2.8 Let $(X, d)$ be a complete multiplicative metric space, $S$ and $T$ be two mappings of $X$ into itself. Suppose that there exist $\lambda \in\left(0, \frac{1}{2}\right)$ and $p, q \in \mathbb{Z}^{+}$such that

$$
d\left(S^{p} x, T^{q} y\right) \leq \max \left\{d^{\lambda}(x, y), d^{\lambda}\left(x, S^{p} x\right), d^{\lambda}\left(y, T^{q} y\right), d^{\lambda}\left(S^{p} x, y\right), d^{\lambda}\left(x, T^{q} y\right)\right\}
$$

for all $x, y \in X$. Then $S$ and $T$ have a unique common fixed point.

Corollary 2.9 Let $(X, d)$ be a complete multiplicative metric space, $S$ and $T$ be two mappings of $X$ into itself. Suppose that there exists $\lambda \in\left(0, \frac{1}{2}\right)$ such that

$$
\begin{aligned}
d(S x, T y) \leq & a_{1} d^{\lambda}(x, y)+a_{2} d^{\lambda}(x, S x) \\
& +a_{3} d^{\lambda}(y, T y)+a_{4} d^{\lambda}(S x, y)+a_{5} d^{\lambda}(x, T y)
\end{aligned}
$$

for all $x, y \in X$. Here $a_{1}, a_{2}, a_{3}, a_{4}, a_{5} \geq 0$ and $0<a_{1}+a_{2}+a_{3}+a_{4}+a_{5} \leq 1$. Then $S$ and $T$ have a unique common fixed point.

Corollary 2.10 Let $(X, d)$ be a complete multiplicative metric space, $S$ and $T$ be two mappings of $X$ into itself. Suppose that there exist $\lambda \in\left(0, \frac{1}{2}\right)$ and $p, q \in \mathbb{Z}^{+}$such that

$$
\begin{aligned}
d\left(S^{p} x, T^{q} y\right) \leq & a_{1} d^{\lambda}(x, y)+a_{2} d^{\lambda}\left(x, S^{p} x\right) \\
& +a_{3} d^{\lambda}\left(y, T^{q} y\right)+a_{4} d^{\lambda}\left(S^{p} x, y\right)+a_{5} d^{\lambda}\left(x, T^{q} y\right)
\end{aligned}
$$

for all $x, y \in X$. Here $a_{1}, a_{2}, a_{3}, a_{4}, a_{5} \geq 0$ and $0<a_{1}+a_{2}+a_{3}+a_{4}+a_{5} \leq 1$. Then $S$ and $T$ have a unique common fixed point.

By taking $S=T$ in Corollaries 2.5-2.10, we have the following results.

Corollary 2.11 Let $(X, d)$ be a complete multiplicative metric space, $S$ be a mapping of $X$ into itself. Suppose that there exists $\lambda \in\left(0, \frac{1}{2}\right)$ such that

$$
d(T x, T y) \leq \phi\left(d^{\lambda}(x, y), d^{\lambda}(x, T x), d^{\lambda}(y, T y), d^{\lambda}(T x, y), d^{\lambda}(x, T y)\right)
$$

for all $x, y \in X$. Then $T$ have a unique fixed point. 
Corollary 2.12 Let $(X, d)$ be a complete multiplicative metric space, $T$ be a mapping of $X$ into itself. Suppose that there exist $\lambda \in\left(0, \frac{1}{2}\right)$ and $p, q \in \mathbb{Z}^{+}$such that

$$
d\left(T^{p} x, T^{q} y\right) \leq \phi\left(d^{\lambda}(x, y), d^{\lambda}\left(x, T^{p} x\right), d^{\lambda}\left(y, T^{q} y\right), d^{\lambda}\left(T^{p} x, y\right), d^{\lambda}\left(x, T^{q} y\right)\right)
$$

for all $x, y \in X$. Then $T$ have a unique fixed point.

Corollary 2.13 Let $(X, d)$ be a complete multiplicative metric space, $T$ be a mapping of $X$ into itself. Suppose that there exists $\lambda \in\left(0, \frac{1}{2}\right)$ such that

$$
d(T x, T y) \leq \max \left\{d^{\lambda}(x, y), d^{\lambda}(x, T x), d^{\lambda}(y, T y), d^{\lambda}(T x, y), d^{\lambda}(x, T y)\right\}
$$

for all $x, y \in X$. Then $T$ has a unique fixed point.

Corollary 2.14 Let $(X, d)$ be a complete multiplicative metric space, $T$ be a mapping of $X$ into itself. Suppose that there exist $\lambda \in\left(0, \frac{1}{2}\right)$ and $p, q \in \mathbb{Z}^{+}$such that

$$
d\left(T^{p} x, T^{q} y\right) \leq \max \left\{d^{\lambda}(x, y), d^{\lambda}\left(x, T^{p} x\right), d^{\lambda}\left(y, T^{q} y\right), d^{\lambda}\left(T^{p} x, y\right), d^{\lambda}\left(x, T^{q} y\right)\right\}
$$

for all $x, y \in X$. Then $T$ has a unique fixed point.

Corollary 2.15 Let $(X, d)$ be a complete multiplicative metric space, $T$ be a mapping of $X$ into itself. Suppose that there exists $\lambda \in\left(0, \frac{1}{2}\right)$ such that

$$
\begin{aligned}
d(T x, T y) \leq & a_{1} d^{\lambda}(x, y)+a_{2} d^{\lambda}(x, T x) \\
& +a_{3} d^{\lambda}(y, T y)+a_{4} d^{\lambda}(T x, y)+a_{5} d^{\lambda}(x, T y)
\end{aligned}
$$

for all $x, y \in X$. Here $a_{1}, a_{2}, a_{3}, a_{4}, a_{5} \geq 0$ and $0<a_{1}+a_{2}+a_{3}+a_{4}+a_{5} \leq 1$. Then $T$ has $a$ unique fixed point.

Corollary 2.16 Let $(X, d)$ be a complete multiplicative metric space, $T$ be a mapping of $X$ into itself. Suppose that there exist $\lambda \in\left(0, \frac{1}{2}\right)$ and $p, q \in \mathbb{Z}^{+}$such that

$$
\begin{aligned}
d\left(T^{p} x, T^{q} y\right) \leq & a_{1} d^{\lambda}(x, y)+a_{2} d^{\lambda}\left(x, T^{p} x\right) \\
& +a_{3} d^{\lambda}\left(y, T^{q} y\right)+a_{4} d^{\lambda}\left(T^{p} x, y\right)+a_{5} d^{\lambda}\left(x, T^{q} y\right)
\end{aligned}
$$

for all $x, y \in X$. Here $a_{1}, a_{2}, a_{3}, a_{4}, a_{5} \geq 0$ and $0<a_{1}+a_{2}+a_{3}+a_{4}+a_{5} \leq 1$. Then $T$ has $a$ unique fixed point.

Competing interests

The authors declare that they have no competing interests.

Authors' contributions

All authors read and approved the final manuscript.

\section{Author details}

${ }^{1}$ Institute of Applied Mathematics, Department of Mathematics, Hangzhou Normal University, Hangzhou, Zhejiang

310036, China. ${ }^{2}$ Department of Mathematics Education and RINS, Gyeongsang National University, Jinju, Korea. 


\section{Acknowledgements}

The authors are grateful to the editor and the reviewer for suggestions, which improved the contents of the article. This work is supported by the National Natural Science Foundation of China (11071169), the Natural Science Foundation of Zhejiang Province (Y6110287) and the Natural Science Foundation of Shandong Province (ZR2013AL015).

\section{Received: 16 May 2015 Accepted: 25 August 2015 Published online: 17 September 2015}

\section{References}

1. Banach, S: Sur les opérations dans les ensembles abstraits et leur application aux équations intégrales. Fundam. Math. 3, 133-181 (1922)

2. Latif, A, Al-Mezel, SA: Fixed point results in quasi-metric spaces. Fixed Point Theory Appl. 2011, Article ID 178306 (2011)

3. $\mathrm{Yu}, J, \mathrm{Gu}, \mathrm{F}:$ Common fixed point theorems for a pair of set-valued maps and two pairs of single-valued maps. J. Hangzhou Norm. Univ., Nat. Sci. Ed. 11(2), 151-156 (2012)

4. Sintunavarat, W, Kumam, P: Common fixed point theorems for a pair of weakly compatible mappings in fuzzy metric spaces. J. Appl. Math. 2011, Article ID 637958 (2011)

5. Singh, SL, Tiwari, BML, Gupta, VK: Common fixed points of commuting mappings in 2-metric spaces and an application. Math. Nachr. 95(1), 293-297 (1980)

6. Popa, V, Imdad, M, Javid Ali, J: Using implicit relations to prove unified fixed point theorems in metric and 2-metric spaces. Bull. Malays. Math. Soc. 33(1), 105-120 (2010)

7. Huang, LG, Zhang, X: Cone metric space and fixed point theorems of contractive mappings. J. Math. Anal. Appl. 332, 1468-1476 (2007)

8. Abbas, M, Jungck, G: Common fixed point results for noncommuting mappings without continuity in cone metric space. J. Math. Anal. Appl. 341, 416-420 (2008)

9. Shi, XT, Gu, F: A new common fixed point for the mappings in cone metric space. J. Hangzhou Norm. Univ., Nat. Sci. Ed. 11(2), 162-168 (2012)

10. Shi, XT, Gu, F: Common fixed point theorem for two pairs of converging commuting mappings in a cone metric space. J. Hangzhou Norm. Univ., Nat. Sci. Ed. 11(5), 433-435 (2012)

11. Altun, I, Erduran, A: Fixed point theorems for monotone mappings on partial metric spaces. Fixed Point Theory Appl. 2011, Article ID 508730 (2011)

12. Aydi, H, Karapinar, E: A Meir-Keeler common type fixed point theorem on partial metric spaces. Fixed Point Theory Appl. 2012, Article ID 26 (2012)

13. Karapinar, E: Generalizations of Caristi Kirk's theorem on partial metric spaces. Fixed Point Theory Appl. 2011, Article ID 4 (2011)

14. Mustafa, Z, Sims, B: Fixed point theorems for contractive mappings in complete G-metric spaces. Fixed Point Theory Appl. 2009, Article ID 917175 (2009)

15. Shatanawi, W: Fixed point theory for contractive mappings satisfying $\Phi$-maps in G-metric spaces. Fixed Point Theory Appl. 2010, Article ID 181650 (2010)

16. Shen, YJ, Lu, J, Zheng, $\mathrm{HH}$ : Common fixed point theorems for converging commuting mappings in generalized metric spaces. J. Hangzhou Norm. Univ., Nat. Sci. Ed. 13(5), 542-547 (2014)

17. Zheng, $\mathrm{HH}$, Shen, YJ, Gu, F: A new common fixed point theorem for three pairs of self-maps satisfying common (E.A) property. J. Hangzhou Norm. Univ., Nat. Sci. Ed. 14(1), 74-78 (2015)

18. Yin, Y, Gu, F: Common fixed point theorem about four mappings in G-metric spaces. J. Hangzhou Norm. Univ., Nat. Sci. Ed. 11(6), 511-515 (2012)

19. Ye, HQ, Lu, J, Gu, F: A new common fixed point theorem for noncompatible mappings of type $\left(A_{f}\right)$ in $G$-metric spaces. J. Hangzhou Norm. Univ., Nat. Sci. Ed. 12(1), 50-56 (2013)

20. Bashirov, AE, Kurplnara, EM, Ozyaplcl, A: Multiplicative calculus and its applications. J. Math. Anal. Appl. 337, 36-48 (2008). doi:10.1155/2008/189870

21. Florack, L, Assen, HV: Multiplicative calculus in biomedical image analysis. J. Math. Imaging Vis. 42(1), 64-75 (2012)

22. Bashirov, AE, Misirli, E, Tandogdu, Y, Ozyapici, A: On modeling with multiplicative differential equations. Appl. Math. J. Chin. Univ. Ser. B 26, 425-438 (2011)

23. Özavşar, M, Çevikel, AC: Fixed point of multiplicative contraction mappings on multiplicative metric spaces (2012). arXiv:1205.5131v1 [math.GM]

24. He, X, Song, M, Chen, D: Common fixed points for weak commutative mappings on a multiplicative metric space. Fixed Point Theory Appl. 2013, Article ID 48 (2013)

25. Abbas, M, Ali, B, Suleiman, Yl: Common fixed points of locally contractive mappings in multiplicative metric spaces with application. Int. J. Math. Math. Sci. 2015, Article ID 218683 (2015). doi:10.1155/2015/218683

26. Kang, SM, Kumar, P, Kumar, S, Nagpal, P, Garg, SK: Common fixed points for compatible mappings and its variants in multiplicative metric spaces. Int. J. Pure Appl. Math. 102(2), 383-406 (2015) 